\title{
Exploring Multi-Surface Interactions in Retail Environments
}

\author{
Sydney Pratte \\ Department of Computer Science \\ University of Calgary \\ Calgary, AB, Canada \\ sapratte@ucalgary.ca \\ Teddy Seyed \\ Department of Computer Science \\ University of Calgary \\ Calgary, AB, Canada \\ teddy.seyed@ucalgary.ca

\section{Frank Maurer} \\ Department of Computer Science \\ University of Calgary \\ Calgary, AB, Canada \\ frank.maurer@ucalgary.ca
}

Permission to make digital or hard copies of part or all of this work for personal or classroom use is granted without fee provided that copies are not made or distributed for profit or commercial advantage and that copies bear this notice and the full citation on the first page. Copyrights for third-party components of this work must be honored. For all other uses, contact the Owner/Author.

Copyright is held by the owner/author(s).

ITS '14, Nov 16-19 2014, Dresden, Germany ACM 978-1-4503-2587-5/14/11.

http://dx.doi.org/10.1145/2669485.2669552

\begin{abstract}
Over the past several years, physical retail outlets have seen a noticeable decline in shoppers, as digital shopping has provided a newer and less costly shopping alternative for consumers. Online digital shopping provides immediate product information but lacks the experience of a physical product. Newer immersive digital shopping environments bring together digital product information with physical products. In this paper we present our early work in exploring the applicability and interaction space of spatially aware multi-surface environments in a retail space. We give an overview on our prototype designed with an industry partner, followed by early feedback on the role of multisurface environments and interactions in the retail space.
\end{abstract}

\section{Author Keywords}

Retail; Multi-surface environments; Device Interaction;

\section{ACM Classification Keywords}

H.5.2 User Interfaces- Input Devices and Strategies; Interaction Styles

\section{Introduction}

As a whole, retail is heavily focused on providing goods and services to a customer, while a retail environment is focused on the overall experience a retailer provides 
to the customer during the process of purchasing goods and services. The retail environment is extremely competitive and has seen increasing competition in several markets, resulting in a large focus on customer experience and engagement instead of past approaches that were more product focused [11]. One approach in enhancing the retail environment is to provide interactive devices to aid customers and enhance their shopping experience, e.g. touch screen information kiosks.

Currently, the biggest threat to the retail environment is online shopping, where information is typically consolidated in a manner that allows consumers to make more informed decisions quickly. Despite the lack of a physical product in online shopping, online shopping is extremely popular and has resulted in retail stores facing "showrooming" challenges, where consumers merely physically browse products and make purchases online.

Research has been exploring interactive devices and technologies as solutions for minimizing "showrooming" [3] by enhancing the retail experience from a customer perspective,e.g. engaging customers with attractive content on displays, in addition to more competitive pricing. Two of the biggest identified technologies for minimizing "showrooming" are based on location and mobile payment [3]. Location is used to allow stores to push promotions to customers when they are near or at a store, while mobile payment allows for significantly less friction when purchasing a product. More recently, retailers have created highly specialized store locations to test new technologies [1]. One example of this type of store is the digital 'lab' store concept launched by Sport Chek ${ }^{1}$.

Given that it is already common for customers to enter stores with interactive devices (e.g. phones and tablets), our goal was to utilize these devices to explore interactions in a spatially aware retail environment. We investigated the role of multi-surface environments in tasks that are common for a typical customer, e.g. browsing for product information, requesting product information, product purchasing. In this work, we present a yearlong collaboration with a retail design partner to explore a multi-surface prototype to create immersive retail environments.

\section{Related Work}

With the surge in popularity of online shopping, retail stores are searching for new and innovative ways to increase the customer's in-store experiences. One approach to improving the retail shopping experience is to integrate physical products and digital environments. To accomplish this integration, various technologies have been introduced to designing retail environments.

An early approach to this problem was a prototype of an interactive system for a retail environment developed by Sukaviriya et al. [11]. The prototype used a steerable projector and user tracking to display product information and track customer interactions. They developed product directories (on a table surface and on a wall), interactive clothing shelves and a mixed media product table. Sukaviriya et al. focused their research on the interactions a user would have while

\footnotetext{
${ }^{1}$ http://www.theglobeandmail.com/technology/tech-news/sport-
chek-sprints-into-digital/article12098883/
} 
moving around a retail space without relying on any other devices [11]. Meschtscherjakov et al. investigated the use of a dynamic map in a retail store [4]. The enhanced store map displayed metric information such as customer "hotspots" and sales ranks and it displayed customer information such as product location and promotions. They discovered that customers respond well to technologies that aid them in familiar tasks such as searching for a specific item and view promotions.

Researchers have also explored the use of mobile device applications with a relationship to a physical space. For example, Newcomb et al. created a mobile application to aid in grocery shopping [5]. They examined how location based information, such as alerts in a specific section, could increase sales. The majority of the research in the retail space, particularly with digital devices, has focused on improving the retai shopping experience by integrating digital information in the physical store, and less on digital devices interacting with each other. A user is interacting with one type of device at a time in much of the research literature presented earlier. Our approach is to bridge the gap between the technologies by introducing a spatially-aware multi-surface environment to the retail space.

Multi-surface Environments (MSE) allow information to be shared among various devices such as tabletops, wall displays, mobile phones and tablets. Spatially aware MSEs use knowledge about location and orientation of devices and people in the space to enhance interactivity. Research in MSEs has been applied and analyzed in different fields. A significant amount of research explores different interactions that can take place in a MSE [8][2][6]. A majority of the research involves supporting collaborative workspaces. An early example is the i-LAND environment [10], which includes an interactive wall display and tabletop and specialized networked chairs. These components come together and allow users to share content across the different devices.

In prior research, we have applied MSEs to the oil and gas domain [9] and in emergency response [7]. The application of MSEs to these very different domains has shown value in multi-device collaboration and data sharing, both of which are very important in the design of our early prototype retail system. For the most part, however, MSEs have been explored in research environments and not in real-world settings.

\section{Designing for the Retail Environment}

To explore the retail environment and build a multisurface environment, we collaborated with an industry design partner in the retail domain, The Brigade Creative Corp., located in Calgary, Canada. From our yearlong collaboration, we created a prototype multisurface solution for the retail environment, with the goal of allowing different retail stakeholders (e.g. customers and employees) to interact with information across multiple devices. The prototype was designed to be scalable and applied to many different retail environments, from smaller shops to larger chain stores. The prototype is intended to aid stakeholders in the traditional retail space and not replace all previous methods. For the design of this prototype, we used a local sports equipment retailer as the initial exploration case. 


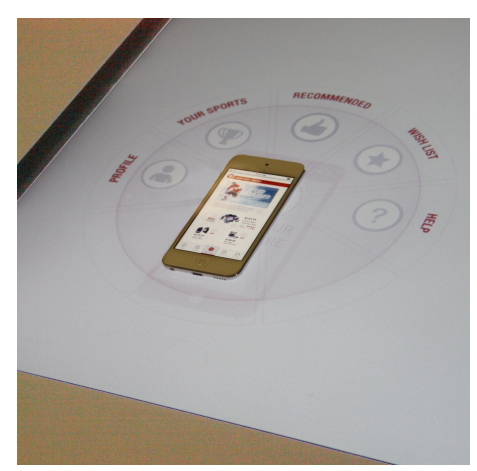

Figure 1. Personalized phone menu that appears when a smart phone device is placed on the tabletop.

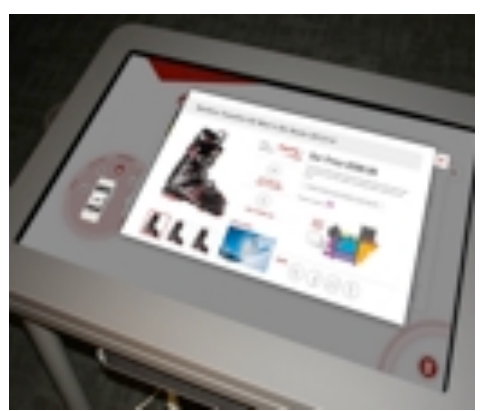

Figure 2. A customer's smart phone receiving product location information.

\section{A RETAIL DESIGN SCENARIO}

In order to illustrate how a retail environment could be supported by multi-surface environments in our prototype, we first describe the design and interaction scenario, as well as personas used with our industry partner (as shown in Figure 3).

\section{STEP 1: A CUSTOMER ENTERS THE STORE}

A customer enters the store with the intention of purchasing a ski boot. As they enter the store, their mobile phone (through a mobile application) notifies them of a type of boot that they might be interested in and directs them to a digital tabletop for more information.

\section{STEP 2: A CUSTOMER USES A TABLETOP}

The customer approaches the tabletop and places their mobile phone on the tabletop and personalized content appears on the tabletop. The customer then views product information, recommendations, ratings and store location, before eventually finding a pair of ski boots. The customer would like a video of the boot being used, and using the tabletop, a "flick" gesture is performed on the tabletop towards a large highresolution wall display, where the video of the boot, as well as other information will be displayed and interacted with.

STEP 3: INTERACTING WITH A WALL DISPLAY

The customer can interact with a 3D image of the boot using gestures in front of the display. Sweeping either arm (left or right) to virtually rotate the boot provides a more complete aesthetic view digitally. The customer then selects the "find in store" option on the wall display by performing a closed fist gesture, causing a map to appear on their smart phone showing the store location of the boot.
STEP 4: MARKETING CONTENT

After locating the boot, the customer heads towards a checkout counter and passes by an advertising display showing new and promotional products. At the same time, a store employee is using a tablet to add a new ad to the displays carousel. The employee "flicks" an ad towards the adverting display, sending it to the display. The customer sees that the ad is a coupon for the ski boots and approaches the display in order to receive it. The customer performs a "flick down" gesture on their smart phone and the ad is now saved to their personal account for checkout.

\section{THE PROTOTYPE}

Based on the previously described scenario, we designed our prototype system with interactions from prior research into multi-surface interactions and gestures [8]. The system consists of several different components: (1) High-resolution wall displays which are used to visually compare products and to display new promotions, (2) A digital tabletop that allows users to search for specific information, (3) Personal devices

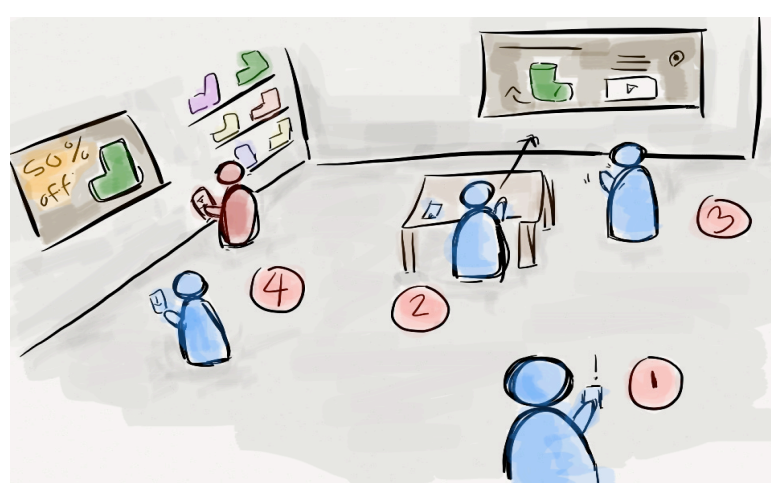

Figure 3. Overview of typical design scenario 


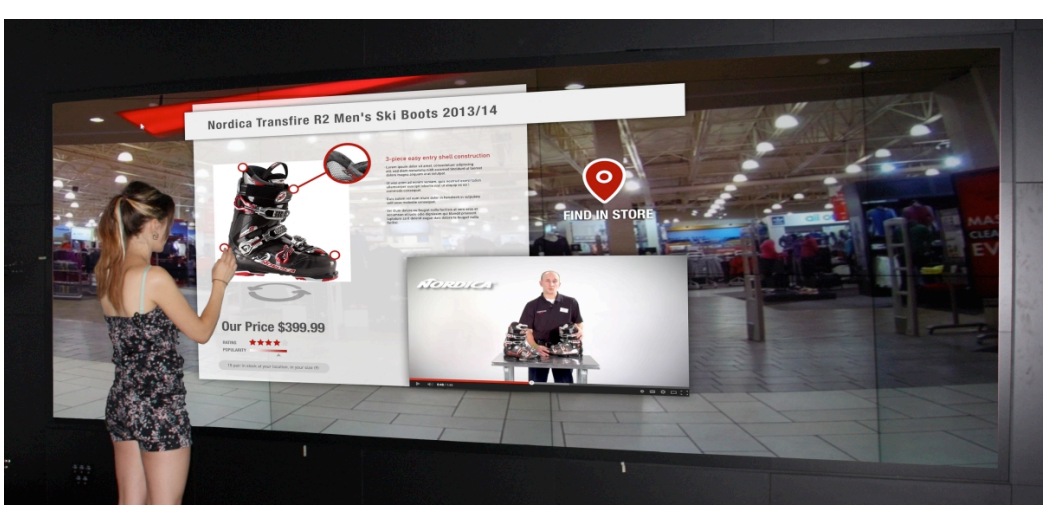

Figure 4. Interacting with a high-resolution display through gestures to find a product in a store.

such as smart phones and tablets that can interact with the environment. The devices and the environment were built using the MSE-API framework ${ }^{2}$.

As described in Step 2, at a digital tabletop, a customer can access product information from an interactive touch wheel menu. This menu contains information based on product categories and featured items. At the tabletop, a user can also access a menu with personal information from their smart phone devices when the phone is physically placed on the tabletop (Figure 1). Additional information also can be sent to a customer's smart phone from the tabletop, for example, a map of the store with a products location (Figure 2 ). The map is sent to the phone either through the interface or by performing a closed fist gesture in front of the wall display. Through the interface and a "flick towards" gesture, the tabletop also sends product information to the wall display, providing a larger view and interaction space. Once the product information is on the wall display, users can see a 360-degree view of a 3D image of the product by rotating the image with either arm (Figure 4).

For employees of the store in this environment, they are able to manipulate different advertising and promotional materials on the different displays by "flicking" towards (to add material) or away from a

${ }^{2}$ MSE-API - https://github.com/ase-lab/MSEAPI-CS display (to remove material). Later, a customer can then save displayed material by performing a downward "flick" on their mobile device, as described in the earlier design scenario.

\section{Initial Feedback from Industry Partner}

For the duration of the design and implementation process of the prototype, we received continual feedback and valuable domain information and insight from our industry partner. This continual feedback helped us create this initial work that will serve as a discussion point for further deployment. Below, we briefly summarize early feedback from initial interviews received through our industry partner.

\section{INITIAL IMPRESSIONS}

When presented with the final prototype for this stage, our industry partner highlighted the result has increased "the possibilities in the retail space", noting that the potential impact on the shopping experience may be greater than initially believed. Feedback from retailers thus far has also mirrored this. We have also observed through feedback, that providing such an environment can also create smarter environments with additional work, such as applying analytics on the location of users in the environment, as well as the type of content they frequently consume with interactions. Overall, we see this as initial evidence that spatially-aware MSEs have potential for enriching retail experiences for both consumers and retailers.

\section{FOCUSING ON THE CUSTOMER}

From the onset of the project the prototype was to be considered a proof of concept and therefore a lot of functionality was still to be built. This was reflected in feedback, however, nearly all this feedback highlighted 
the need for even more interactions between customer devices and the environment. This highlights the need for even more focus on customers in designing retail multi-surface environments.

\section{Conclusion and Future Work}

In this paper, we present our initial work on a multisurface prototype designed specifically for the retail domain. The interactions implemented were based upon prior research, as well as experience in several other domain areas. As we have shown in this initial work, there is interest and value for the retail domain with multi-surface environments and interactions.

The next step in our research is to deploy the prototype in a retail environment and conduct user studies to evaluate the impact on customers (e.g. user engagement, satisfaction) as well as retailers (e.g. reduced showrooming, impact on sales). It is our hope that this initial work will create a greater interest in adapting a multi-surface environment in the retail domain and create richer and more engaging retail experiences.

\section{References}

[1] Burke, Raymond R. Virtual Shopping: Breakthrough in Marketing Research, Harvard Business Review (1996).

[2] Dachselt, R., and Buchholz, R. Natural throw and tilt interaction between mobile phones and distant displays. Ext. Abstracts CHI 2009, Boston, MA, USA (2009), 3253-3258.

[3] iQmetrix. "In-Store Technology: Delivering the Shopping Experience Customers Want."

http://www.iqmetrix.com/documents/XQ/Customer-

Experience_Whitepaper.pdf
[4] Meschtscherjakov, A., Reitberger, W., Lankes, M., and Tscheligi, M. In Proc. UbiComp 2008, ACM Press (2008), 336-339.

[5] Newcomb, E., Pashley, T., and Stasko, J. Mobile computing in the retail arena. In Proc. CHI 2003, ACM Press (2003), 337-344.

[6] Rekimoto, J. Pick-and-drop: a direct manipulation technique for multiple computer environments. In Proc. UIST 1997, Banff, Alberta, Canada (1997), 31-39.

[7] Rodrigues, F. M., Seyed, T., Chokshi, A., and Maurer, F. Gesture Design and Feasibility in Emergency Response Environments. In Proc. CHI 2014, Toronto, Canada (2014).

[8] Seyed, T., Burns, C., Costs Sousa, M., Maurer, F., and Tang, A. Eliciting usable gestures for multi-display environments. In Proc. ITS 2012, ACM Press (2012), 41-50.

[9] Seyed, T., Costs Sousa, M., Maurer, F., and Tang, A. SkyHunter: a Multi-Surface Environment for Supporting Oil and Gas Exploration. In Proc. ITS 2013, ACM Press (2013), 15-22.

[10] Streitz, N., Geibler, J., Holmer, T., Konomi, S., Muller-Tomfelde, C., Reischi, W., Rexroth, P., Seitz, P. and Steinmetz, R. i-LAND: an interactive landscape for creative innovation. In Proc. CHI 1999, ACM Press (1999), 120-127.

[11] Sukaviriya, N., Podlaseck, M., Kjeldsen, R., Levas, A., Pingali, G., and Pinhanez, C. Augmenting a Retail Environment Using Steerable Interactive Displays. Ext. Abstracts CHI 2003, ACM Press (2003), 978-979. 